\title{
Sellar plasmacytoma presenting with symptoms of anterior pituitary dysfunction
}

\author{
Ana G Ferreira', Tiago N Silva', Henrique V Luiz', Filipa D Campos², Maria C Cordeiro' \\ and Jorge R Portugal1
}

${ }^{1}$ Endocrinology and Diabetes Department and ${ }^{2}$ Hemato-Oncology Department, Garcia de Orta Hospital, Almada, Portugal
Correspondence should be addressed to A G Ferreira

Email

adgonfer@gmail.com

\section{Summary}

Sellar plasmacytomas are rare and the differential diagnosis with non-functioning pituitary adenomas might be difficult because of clinical and radiological resemblance. They usually present with neurological signs and intact anterior pituitary function. Some may already have or eventually progress to multiple myeloma. We describe a case associated with extensive anterior pituitary involvement, which is a rare form of presentation. A 68-year-old man was referred to our Endocrinology outpatient clinic due to gynecomastia, reduced libido and sexual impotence. Physical examination, breast ultrasound and mammography confirmed bilateral gynecomastia. Blood tests revealed slight hyperprolactinemia, low testosterone levels, low cortisol levels and central hypothyroidism. Sellar MRI showed a heterogeneous sellar mass $(56 \times 60 \times 61 \mathrm{~mm})$, initially suspected as an invasive macroadenoma. After correcting the pituitary deficits with hydrocortisone and levothyroxine, the patient underwent transsphenoidal surgery. Histological examination revealed a plasmacytoma and multiple myeloma was ruled out. The patient was unsuccessfully treated with radiation therapy (no tumor shrinkage). Myeloma ultimately developed, with several other similar lesions in different locations. The patient was started on chemotherapy, had a bone marrow transplant and is now stable (progression free) on lenalidomide and dexamethasone. The presenting symptoms and panhypopituitarism persisted, requiring chronic replacement treatment with levothyroxine, hydrocortisone and testosterone.

\section{Learning points:}

- Plasmacytomas, although rare, are a possible type of sellar masses, which have a completely different treatment approach, so it is important to make the correct diagnosis.

- Usually, they present with neurological signs and symptoms and a well-preserved pituitary function, but our case shows that anterior pituitary function can be severely compromised.

- Making a more extensive evaluation (clinical and biochemical) might provide some clues to this diagnosis.

\section{Background}

A solitary plasmacytoma is a possible presentation form of multiple myeloma. Sellar plasmacytomas are very uncommon and can be easily mistaken with a nonfunctioning pituitary adenoma, because of clinical and radiological resemblance (1). Most cases described in the literature presented with neurological symptoms (2) and normal (or slightly affected) anterior pituitary function (3). We describe a case of sellar plasmacytoma that presented with symptoms caused by anterior pituitary hormonal disturbance and had an extensive anterior pituitary involvement, which is a rare form of presentation.

\section{Case presentation}

A 68-year-old man was referred to our Endocrinology outpatient clinic for evaluation of gynecomastia. 
The patient noticed bilateral breast enlargement and pain on the right breast 3 months earlier and also complained of decreased libido and sexual impotence. He had no other complaints, namely galactorrhea, diplopia or new visual disturbances and headaches.

His past medical history included hypertension, hyperlipidemia, glaucoma (left eye), right eye prosthesis due to trauma (total blindness from that side) and a left forearm fracture (traumatic, occurred 15 years before, unlikely related to osteoporosis). He was on lisinopril $(20 \mathrm{mg})+$ hydrochlorothiazide $(12.5 \mathrm{mg})$ and bimatoprost+timolol eye drops. The patient denied alcohol abuse and illegal drug use. The remaining medical history and family history were unremarkable. Physical examination revealed bilateral painful gynecomastia, more pronounced on the right side, no milk, blood or any other fluid were noticed spontaneously or after nipple expression. Testicular volume $>15 \mathrm{~mL}$, no testicular masses. Neurological examination was unremarkable and no other relevant findings were identified.

\section{Investigation}

Breast ultrasound and mammography were performed, confirming bilateral dendritic gynecomastia, more pronounced on the right side, without suspicious nodules.

His initial blood tests revealed panhypopituitarism: slight hyperprolactinemia, low cortisol levels,

Table 1 Initial laboratory assessment.

\begin{tabular}{l}
\hline Laboratory tests \\
\hline Hemoglobin \\
Creatinine \\
AST \\
ALT \\
GGT \\
ALP \\
Total bilirubin \\
Prolactin \\
FSH \\
LH \\
Total testosterone \\
Free testosterone \\
Estradiol \\
TSH \\
Free T4 \\
Total T3 \\
IGF-1 \\
Cortisol \\
ACTH \\
$\beta$ ChCG
\end{tabular}

hypogonadotrophic hypogonadism and central hypothyroidism (Table 1). No posterior involvement was apparent (clinical or biochemical).

We performed an ACTH stimulation test at 08:00 h that was suggestive of adrenal insufficiency (peak cortisol: $16.1 \mu \mathrm{g} / \mathrm{dL}$ at $60^{\prime}$; normal response: $>18 \mu \mathrm{g} / \mathrm{dL}$ ).

The sellar magnetic resonance imaging (MRI) (Fig. 1) showed a heterogeneous mass located at the middle cranial fossa, centered at the clivus, with $56 \times 60 \times 61 \mathrm{~mm}$, with marked contrast enhancement, hypointense on T2-weighted imaging with some hyperintense areas. Anteriorly, the mass invaded the sphenoid sinus and posterior ethmoidal air cells. Laterally, it invaded both cavernous sinus, more extensively the left one. The mass was indistinguishable from the pituitary gland. Pituitary stalk was markedly enlarged, from mass extension. No optic chiasma compression or diencephalic extension was present. Posteriorly, it extended to the pons, slightly compressing it. Inferiorly, it extended to the pterygoid processes. The radiologist concluded that it was most likely an invasive macroadenoma, but the differential diagnosis included plasmacytoma and a tumor from sphenoid or ethmoid bone.
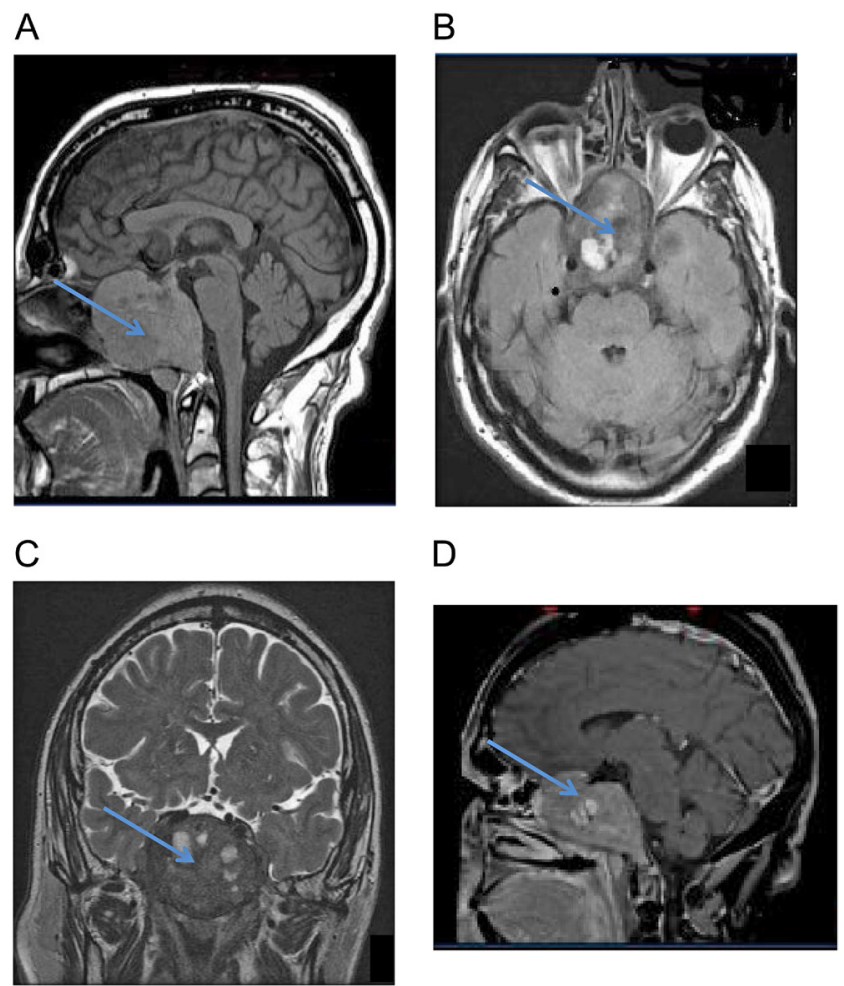

Figure 1

Sellar MRI showing a volumous tumor (arrow). (A) T1, sagittal; (B) T2, axial; (C) T2, coronal; (D) sagittal, post gadolinium. 
A

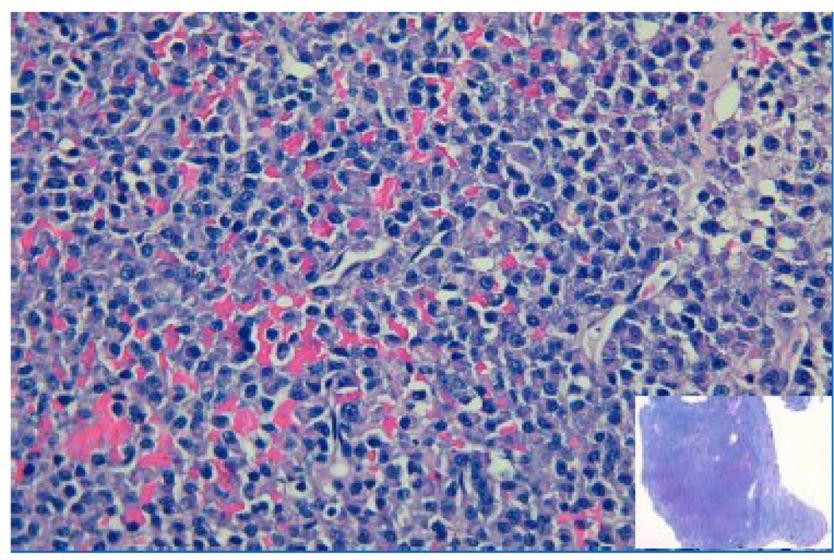

B

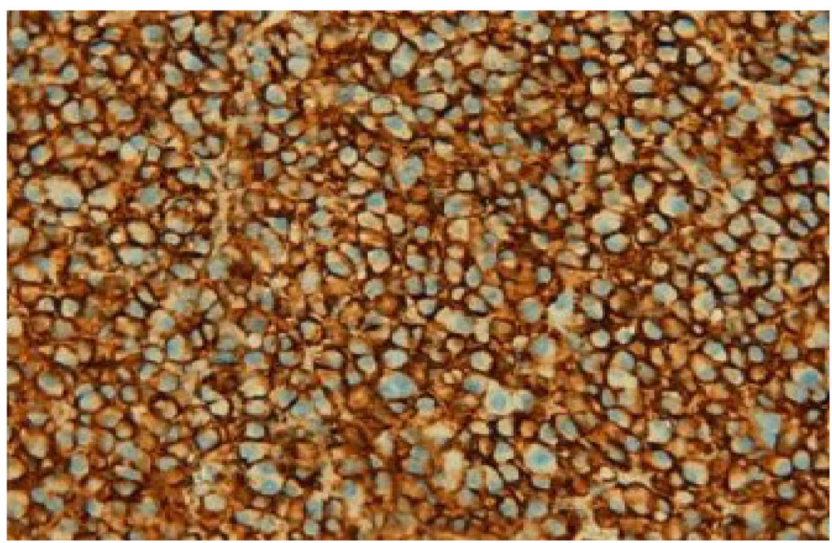

C

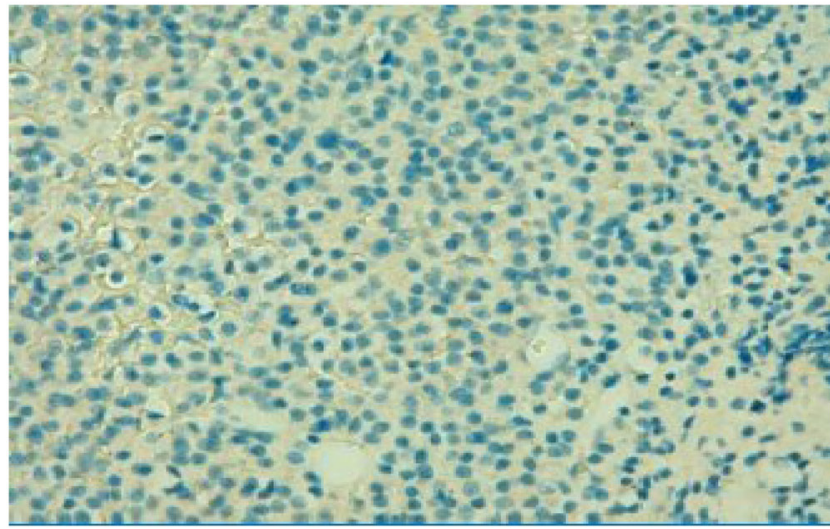

Figure 2

Histological examination. (A) Hematoxylin-eosin (400 and 40x); (B) CD138 immunohistochemical staining (400x); (C) CK AE1/AE3 immunohistochemical staining (400x).
We performed a left eye visual field examination, with no relevant findings apart from those related to glaucoma.

\section{Treatment}

The patient was treated with hydrocortisone $20 \mathrm{mg}$ /day and later with levothyroxine $25 \mu \mathrm{g} /$ day and subsequently underwent transsphenoidal surgery. During the procedure, he had significant bleeding (hypervascular mass) requiring blood transfusions, and surgeons were only able to remove a small part of the tumor. Histological examination revealed a tumor composed of plasma cells, CD138+, CK AE1/AE3 neg, Ki67 20\%, compatible with plasmacytoma (Fig. 2).

At this point, we evaluated the patient for the presence of multiple myeloma. We found a slightly raised 凶2-microglobulin, a positive Bence Jones protein (kappa light chains) and a very discrete monoclonal peak of IgG kappa, normal calcium levels, normal total light chains and elevated free kappa light chains. There were no lytic bone lesions on the skeletal survey (X-rays) or evidence of any other end-organ damage. Myelogram and bone marrow biopsy had no clonal plasma cells.

So far, the patient seemed to have an isolated sellar plasmacytoma, and we decided to treat him with localized radiation therapy (50Gy) during 4 weeks, unsuccessfully (the tumor was not reduced in size). He ended up progressing to multiple myeloma about 6 months after surgery: kappa light chains started to raise, mild renal failure and normochromic normocytic anemia developed and the FDG-PET-CT scan showed evidence of active disease in multiple sites (several costal arches, the most significant lesions at the 11th and 12th right costal arches; sternum; L4 vertebrae; right breast) - Fig. 3. Still no medullary involvement.

The patient was started on chemotherapy (cyclophosphamide, bortezomib and dexamethasone) and proposed for a bone marrow auto-transplant. After completing 4 cycles (once a month), there was a partial response, with normalization of kappa light chains, negative Bence Jones protein and metabolic improvement of the lesions detected on FDG-PET scan.

The transplant was performed about 1 year after surgery, with a post-transplant infection by varicelazoster (left auricular canal). However, 3 months after 
A
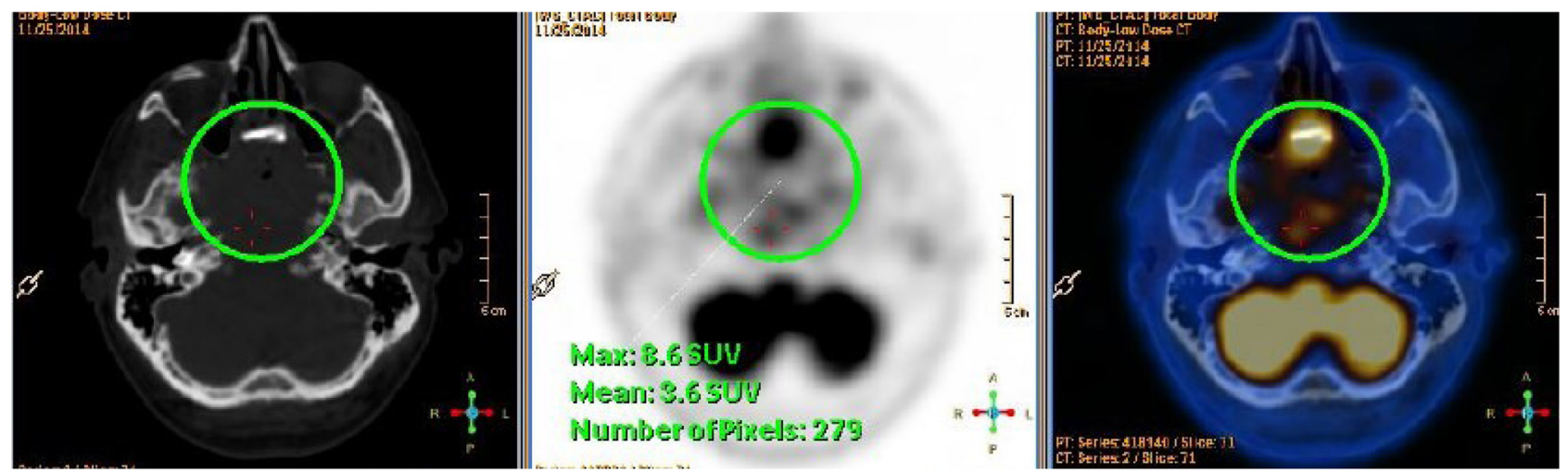

\section{B}
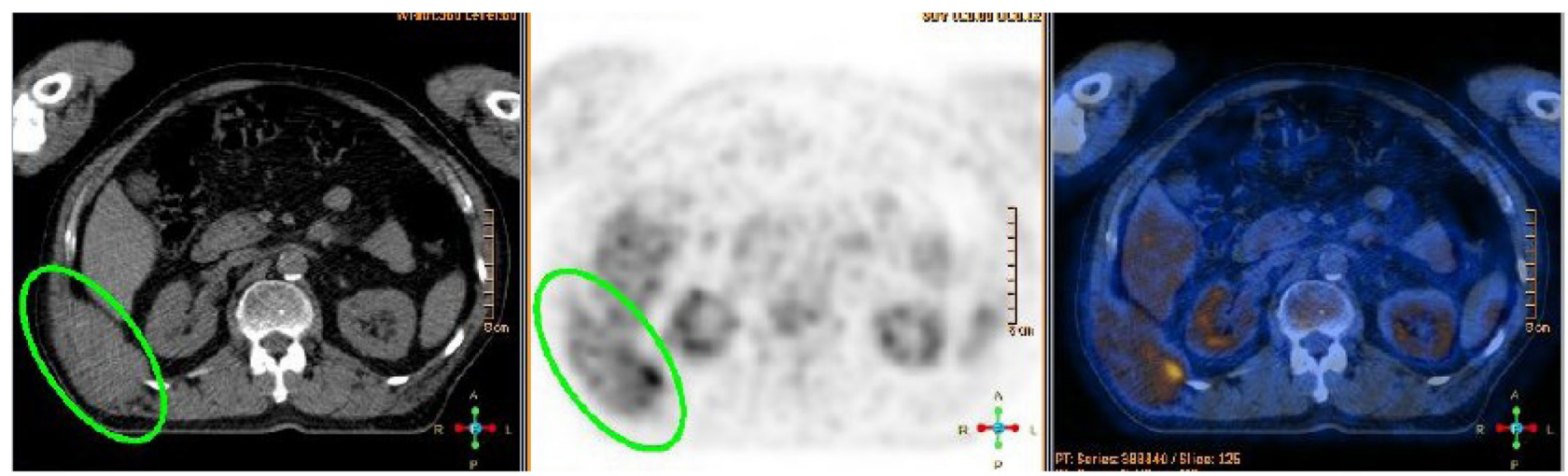

C
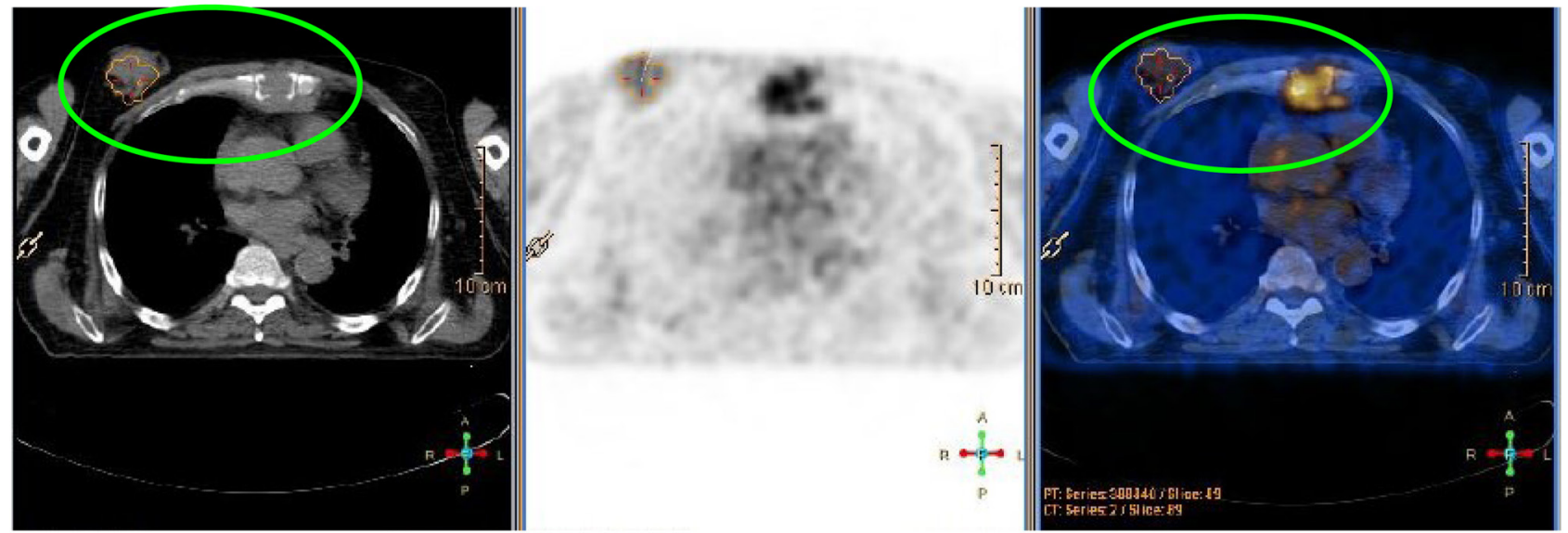

Figure 3

FDG-PET-CT images. (A) Sellar plasmacytoma; (B) lesions at the 11th and 12th right costal arches; (C) lesions at the sternum and right breast.

the transplant, the scans showed that the disease was progressing again, with half of the bone lesions showing increased metabolic activity, so the patient was started on lenalidomide $25 \mathrm{mg}$ and dexamethasone $40 \mathrm{mg}$ (28 day cycles). He did not tolerate the treatment well, with oral candidiasis and prostration, so the dose was reduced. After 4 cycles, there was a slight reduction in size of the sellar mass, but a heterogeneous response of all other lesions, with new small ones at the costal arches and sternum. 


\section{Outcome and follow-up}

At the time this paper was written, the patient had completed 9 cycles of lenalidomide and dexamethasone and the disease is still extensive, but stable.

The asymmetrical gynecomastia and panhypopituitarism persisted. Levothyroxine was raised to $75 \mu \mathrm{g} /$ day and corticosteroid replacement therapy was stable at $30 \mathrm{mg} /$ day of hydrocortisone. Testosterone replacement therapy was also started (testosterone enanthate $250 \mathrm{mg}$ every 4 weeks).

\section{Discussion}

Extramedullary plasmacytomas are very rare, representing less than 3\% of all plasma cell neoplasms (4). Sellar plasmacytomas (specially as the first clinical manifestation of myelomatous disease - (5)) are even more infrequent, with only a few cases described so far. There are less than a hundred known cases. Lee and coworkers (6) performed a recent review of 70 cases of sellar and parasellar plasmacytomas (including 5 new cases reported by the authors). Another 11 different cases have also been included by Joukhadar and coworkers in their previous review (7).

In most of these cases, patients presented with headaches and visual disturbances (mainly diplopia, but also visual loss or blurred vision) $(1,6,8)$. Cranial nerve involvement was frequent, mostly III, IV and VI nerves (1, 2, 8), but also II, V, VII and VIII $(1,7,9)$.

Data regarding the anterior pituitary function in patients with sellar plasmacytomas are not always reported, but it is usually well preserved $(3,5,6,7,8,9)$, unlike our case. Lee and coworkers (6) present 1 patient with hypogonadotrophic hypogonadism and 1 patient with hyperprolactinemia, without hypopituitarism. In their review, they mention 38\% (of 26 patients with reported prolactin levels) with hyperprolactinemia and $15 \%$ (of 33 patients with reported endocrine evaluation) with hypopituitarism (including their own). The hypopituitarism cases included a patient reported by Sinnot and coworkers (5) with inappropriately normal LH and FSH for a menopausal woman (and minimally elevated prolactin levels); a patient described by Joukhadar and coworkers (7) with central hypothyroidism (and hyperprolactinemia); a patient described by Udiawar and coworkers (3) who complained of loss of libido and reduced frequency of erection that turned out to have low testosterone levels, with normal FSH and LH levels (although the authors interpreted this result as possible hypogonadotrophic hypogonadism, they did not perform a LHRH stimulation test to confirm it, at least it is not described in their paper). Less robust cases reviewed included a patient with slightly impaired cortisol response to insulin-induced hypoglycemia and a borderline low response of TSH to TRH stimulation test and a case of isolated low testosterone levels with no mention to FSH and $\mathrm{LH}$.

Therefore, it seems that our case is probably the one with the most pronounced clinical and biochemical anterior pituitary involvement related to a sellar plasmacytoma described so far. The absence of posterior deficits is also a feature of this case and, to the best of our knowledge, there is no other case described in the literature with posterior involvement.

Another particular aspect of our case is that it presented with no neurological complaints, although the absence of visual disturbances can actually be related to the fact that the patient had already a very significant visual compromise.

Sellar plasmacytomas may be easily mistaken with other sellar masses in imaging studies, so the definite diagnosis is histological $(1,8)$. Some features described in the literature that are commonly related to plasmacytomas include large size, extension to adjacent areas and bone destruction (sellar floor, clivus) $(6,7)$. In their review, Lee and coworkers (6) report a tendency for homogeneous contrast enhancement, with iso or hypointense lesions on T1-weighted imaging and hyperintense on $\mathrm{T} 2$, although they recognize when discussing this issue that plasmacytomas are usually homogeneous masses, iso or hyperintense on T1-weighted imaging and hypointense on T2.

The 2009 working group of UKMF Guidelines (10) recommends that solitary extramedullary plasmacytomas should be treated with radiation therapy, which usually allows a good local control of the disease (unlike our patient). Most patients described in the literature with local disease were treated with radiotherapy, many after surgery (only a few with gross total resection), with good response rates, and chemotherapy was mainly used for patients with systemic disease $(2,6,7)$. Only a few patients underwent a bone marrow transplant $(2,6)$. Lee and coworkers (6) in their case series describe a $38 \%$ progression rate to multiple myeloma. The overall survival is very good, although less if progression occurs (6).

In conclusion, sellar masses may represent a true diagnostic challenge. Plasmacytomas in this location are rare, but should be included in the differential diagnosis because they have a specific therapeutic approach. There is no single characteristic (clinical, biochemical or 
imagiological) that allows us to easily make this diagnosis, although plasmacytomas are frequently large masses, with great extension to the surrounding structures, presenting with intact anterior pituitary function (which was not found in our case) and neurologic compromise. In fact, to the best of our knowledge, we report the most extensive anterior pituitary compromise described in the literature so far in a patient with a sellar plasmacytoma. Longterm follow-up is required to evaluate for progression to multiple myeloma, and adequate treatment is mandatory.

\section{Declaration of interest}

The authors declare that there is no conflict of interest that could be perceived as prejudicing the impartiality of the research reported.

\section{Funding}

This work did not receive any specific grant from any funding agency in the public, commercial or not-for-profit sector

\section{Patient consent}

Written informed consent was obtained from the patient described in this article for publication of the article and images therein.

\section{Author contribution statement}

Tiago N Silva: article reviewer. Henrique V Luiz: article reviewer and patient's Endocrinologist. Filipa D Campos: article reviewer and patient's Haematologist. Maria C Cordeiro: article reviewer. Jorge R Portugal: article reviewer. This paper was written with permission and revision from the patients' physicians (who are also authors, as stated earlier).

\section{References}

1 Jiang CZ, Lin QS, Wu XY, Wang CY \& Kang DZ 2014 Sellar solitary plasmacytoma progressing to multiple myeloma: a case report and literature review. Medicine 93 1-6. (https://doi.org/10.1097/01. md.0000464196.31017.33)

2 Khan IS, Javalkar V, Thakur JD \& Nanda A 2012 Intrasellar plasmacytoma: an illustrative case and literature review. Journal of Clinical Neuroscience 19 210-213. (https://doi.org/10.1016/j. jocn.2011.07.009)

3 Udiawar M, Bejnariu C \& Davies S 2012 Metastatic haematological malignancy presenting as a sellar mass. BMJ Case Reports 2012 bcr0320126050. (https://doi.org/10.1136/bcr-03-2012-6050)

4 Gerry D \& Lentsch EJ 2013 Epidemiologic evidence of superior outcomes for extramedullary plasmacytoma of the head and neck. Otolaryngology: Head and Neck Surgery 148 974-981. (https://doi. org/10.1177/0194599813481334)

5 Sinnot BP, Hatipoglu B \& Sarne DH 2006 Intrasellar plasmacytoma presenting as a non-functional invasive pituitary macro-adenoma: case report \& literature review. Pituitary 9 65-72. (https://doi. org/10.1007/s11102-006-8281-9)

6 Lee J, Kulubya E, Pressman BD, Mamelak A, Bannykh S, Zada G \& Cooper O 2017 Sellar and clival plasmacytomas: case series of 5 patients with systematic review of 65 published cases. Pituitary $\mathbf{2 0}$ 381-392. (https://doi.org/10.1007/s11102-017-0799-5)

7 Joukhadar R \& Chiu K 2012 Sellar plasmacytomas: a concise review. Pituitary 15 146-149. (https://doi.org/10.1007/s11102-011-0352-x)

8 Soejbjerg A, Dyve S, Baerentzen S, Thorsell G, Poulsen PL, Jorgensen JL \& Kampmann U 2016 The solitary sellar plasmacytoma: a diagnostic challenge. Endocrinology, Diabetes and Metabolism Case Reports 2016 160031. (https://doi.org/10.1530/EDM-16-0031)

9 Yaman E, Benekli M, Coskun U, Sezer K, Ozturk B, Kaya AO, Yildiz R, Uluoglu O \& Buyukberber S 2008 Intrasellar plasmacytoma: an unusual presentation of multiple myeloma. Acta Neurochirurgica 150 921-924. (https://doi.org/10.1007/s00701-008-0012-8)

10 Hughes M, Soutar R, Lucraft H, Owen R \& Bird J Guidelines on the diagnosis and management of solitary plasmacytoma of bone, extramedullary plasmacytoma and multiple solitary plasmacytomas: 2009 update. British Committee for Standards in Haematology, London, UK, 2009.

Received in final form 24 October 2017

Accepted 3 November 2017 